\title{
Analysis of Power Saving Routing Protocol for Wireless
}

\author{
Sasikumar $\mathrm{P}$ \\ Assistant professor \\ School of Electronics Engineering \\ VIT University
}

\author{
Mathew Abraham Roy \\ UG Scholar \\ School of Electronics Engineering \\ VIT University
}

\begin{abstract}
In Wireless nodes power saving is an important issue always been raised due to limited energy stored in them. For saving power various methods are implemented, using routing protocols, sleep mode operations and efficient processing algorithms. In this work, we used Network Simulator-2 to generate a wireless adhoc network and packets are routed through them using the AODV (ad-hoc on-demand distance vector) routing protocol. The Trace file is analyzed to determine the energy used by each of the nodes for routing the generated packets through the network. The most of the energy consumed in ad-hoc network is found to be the transmission of packets between the nodes. The goal of the project is to increase the energy efficiency of AODV routing protocol by incorporating Transmission Power Control Technique. This method calculates the distance between a transmitting node and a receiving node, and selects transmission power with respect to the distance between the transmitter and receiver.

The simulations are done for single source, multiple sources as well as for light and dense network. The comparison of output files for each of the scenarios shows significant reduction of energy consumption in the new proposed method than in AODV. The results of this project are very significant as it can extend the life of wireless ad-hoc network considerably and can be introduced to other routing protocols as well.
\end{abstract}

\section{Categories and Subject Descriptors}

C.2.1 [Network Architecture and Design]: Wireless communication; C.2.5 [Computer-Communication Net-works]: Local and Wide-Area Networks; I.6 [Computing Methodologies]: Simulation and Modeling

\section{General Terms}

Algorithms, Experimentation, Performance

\section{Keywords}

Ad-hoc Networks, Power Saving routing protocols, AODV, COMPOW, and CMMBCR Protocol.

\section{INTRODUCTION}

The Energy conservation is major constraint for a wireless ad-hoc network as they are powered by small battery. Various methods have been proposed to increases the energy conservation. Some are Topology control, power aware routing and sleep management. In topology control as in [4], a node decides to relay through other node when less power is used. In power aware routing, transmission power is set depending on the distance of the receiver and transmitter [6]. The life expectancy of the network can be increased by switching off nodes with less battery remaining. When the battery remaining in a node goes below a certain threshold the node is put to sleep and a route error packet is broadcasted. This initializes a new route discovery process which finds another path for the packet to be routed [4]. Several modifications are done to AODV on this basis. Energy saving (ES)-AODV introduces power control mechanism on the MAC layer, combining link node and overall energy costs with the remaining energy to select route. The effects of reduced transmission are listed out in [6]. The capacity of a wireless adhoc network is depends on the jamming of the nodes in the network, which in turn depends on the power at which these nodes transmit. By reducing the power at a node, the interference range of that node is reduced and hence the capacity of the network as a whole is increased.

\subsection{Applications of Ad-Hoc Networks}

- Nodes are embedded in bridges when you pour concrete. The nodes are fitted with salt concentration detector. This helps in detecting the weakened Portions of concrete and corrosion of steel rebar. The motes transmit their readings to a collection centre from where the readings are retrieved monthly.

- $\quad$ Nodes could be attached to the water or power meters in a neighborhood. The node log power and water consumption of a customer and transmits to a collection center.

- A biologist wishing to learn about the behavior of wild animals in their natural surrounding could fit a wireless sensor to the animal. Certain parts of the jungle can have collection centers. When the animal wanders into these zones the collected data could be transmitted.

- $\quad$ Nodes placed on a highway could be equipped with sensors to detect traffic flow and could help police control traffic flow and immediately be notified in case of an accident occurs.

- They can also be used in warfare to sense battle field conditions. It can be fitted with a vibration sensor and a GPS unit. The nodes are distributed across a field where enemy maybe expected to pass through. When an enemy truck goes nearby, the vibrations are picked up by the sensor, and the nodes transmit the information to a collection center, which has either a wired link or satellite link to the control center. The officer could have a UAV make sure it's an enemy truck and have it drop bombs. 


\section{THEORETICAL BACKGROUND 2.1 The Impact of Transmission Power Control}

Transmit power control is important in any wireless networks for the reasons: (i) considering battery life, and (ii) burden on traffic carrying capacity of the network [8].

For the first point, note that there is no need for $\mathrm{N} 1$ in Figure 1 to broadcast at $30 \mathrm{~mW}$ (range at $30 \mathrm{~mW}=250 \mathrm{~m}$ ) to send a packet to the neighboring $\mathrm{N} 2$, since $\mathrm{N} 2$ is within range even at $1 \mathrm{~mW}$. Thus it can save on battery power if transmission is done at

$1 \mathrm{~mW}$.

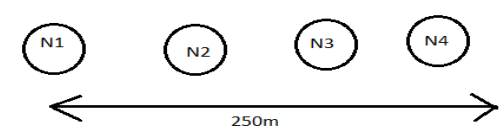

Figure 1. Interference between neighboring nodes

For the second point, suppose that in the same figure, N3 also wishes to broadcast a packet at the same time to N4 at $1 \mathrm{~mW}$. If $\mathrm{N} 1$ broadcasts at $1 \mathrm{~mW}$ to $\mathrm{N} 2$, then both transmissions can be successfully received simultaneously, since neither is N2 in the range of its interferer $\mathrm{N} 3$ (for its reception from N1), nor is $\mathrm{N} 4$ in the range of its interferer N1. However, if an N1 broadcast at $30 \mathrm{~mW}$, then that interferes with N4's reception from N3, and so only one packet, from N1 toN2, is successfully transmitted. Thus, power control can enhance the traffic carrying capacity.

When a node's radio transmission power is controllable, transmitting range and the number of its immediate neighbors to reach the destination nodes are adjustable [8]. Higher values of transmission power increases the transmission range and reduces the hop count to the destination, lesser transmission power makes the topology partitioning resulting in high end-to-end delay due to a larger hop count. Also if the transmission power is controllable, it may be more energy efficient to transmit packets using intermediate nodes because the required transmission power, $p$, to communicate between two nodes has super-linear dependence on distance, $d$, i.e.,. $p(d) \propto(d)^{2}$

The transmission power control [7] also helps to deal with irregular distribution of nodes over an area. If the inter-nodal distances are different, there may exist some nodes that are closely placed while there may be others that have high internodal separation. If a constant transmission power is used, a lot of energy gets wasted when nodes with very less separation transmits at high power. This also prevents other nodes close by from transmitting packets.

Basically two propagation models exist:-

- $\quad$ Friss Transmission model

- $\quad$ Two Ray Propagation model

$$
P_{r}=P t \times\left(\frac{\lambda}{4 \pi d}\right)^{n} \times G_{t} \times G_{r}
$$

Where $P_{r}=$ received power, $P t=$ transmitted power, $d=$ distance between the transmitter and the receiver, $G_{t}=$ gain of transmitting antenna, $G_{r}=$ gain of receiving antenna, $n=2$ for friss transmission, and $n=4$ for two ray propagation

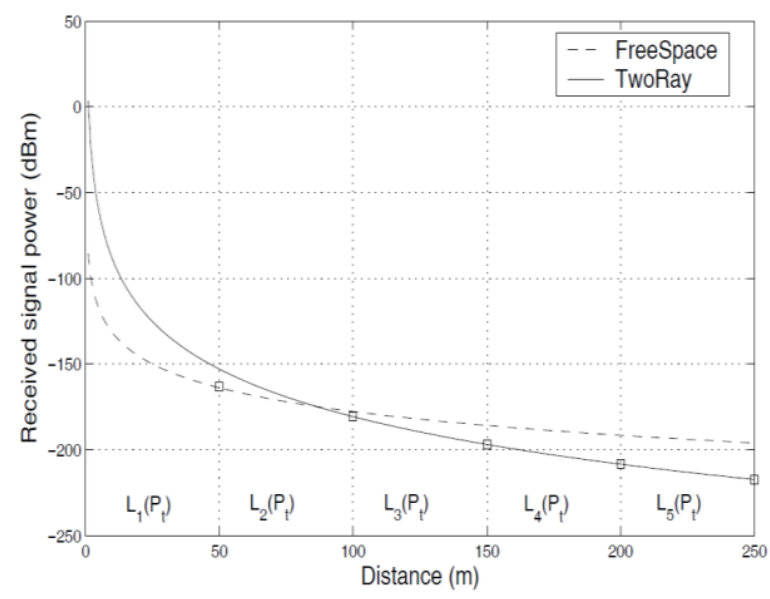

Transmission power level Li(Pt) based on received signal power

Figure 2. Transmission power Vs Received signal power

Various methods have been proposed to determine the transmission power to be used for inter-nodal communication. Some of them are described below.

- $\quad P_{t}$ can be set by calculating it with respect to received power of hello packets broadcasted. This method is not followed in this project since its broadcasting hello packets at regular intervals can be considered a waste of energy. And so it's been disabled. Route Request and Route Error are the only broad casted packets.

- Another proposed method is the determination of required transmission power level by comparing the degree of power attenuation with multiple predefined threshold values required to setup transmission power level.

\section{METHODOLOGIES}

\subsection{Smallest Common Power (COMPOW) protocol}

This protocol provides to maintain bi-directionality between paired (any two) of communicating nodes in a MANET [3]. This is achieved by having all the nodes in the MANET maintain a same transmission power level $\left(P_{i}\right)$. If $P_{i}$ is too low, a node can communicate to limited number of nodes in a MANET. If $P_{i}$ is very high, node will communicate with all other nodes without the help of neighbor nodes but consumes more energy. Hence a node can directly or indirectly reach the entire MANET with a smaller $P_{i}$. Therefore, the optimum power level $\left(P_{i}\right)$ is the smallest power level at which all the nodes in the entire network is connected. In COMPOW, it is assumed that the transmission power levels cannot be arbitrarily adjusted but instead it must be selected among a small number of discrete power levels $(P l$, $P 2, \ldots$ P $\max )$. Different power levels result in different node 
connectivity since they cover different radio transmission ranges. Each node maintains a routing table as in table-driven routing mechanism, but one for each power level (RTP1, RTP $2, \ldots$, RTPmax). The number of entries in RTPi, denoted as $|R T P i|$, means the number of reachable nodes at Pi. This includes directly connected nodes as well as indirectly connected nodes via intermediate nodes. By exchanging these routing tables, nodes find the minimal $\mathrm{Pi}$ that satisfies $|R T P i|=n$ for all nodes, where $\mathrm{n}$ is the total number of nodes in the MANET. Extended solutions are also discussed in for the case where there are many discrete power levels and where the latency involved with switching power levels is not negligible.

\subsection{Conditional Max-Min Battery Capacity Routing (CMMBCR) Protocol}

[6] The CMMBCR protocol uses the concept of a threshold to maximize the lifetime of each node and fair usage of battery energy. If all nodes in some possible routes between a sourcedestination pair battery have larger energy remaining, than the threshold, the min-power route among those routes is selected. If all possible routes have nodes with lower battery capacity than the threshold, the max-min route is selected. However, the threshold value is fixed leading to a simpler design. The authors of this protocol proposed an interesting performance metric for measuring the energy balance: expiration sequence, defined as the sequence of times when mobile nodes exhaust their battery capacity. Traditional metrics for energy balance are variation of remaining battery capacity, ratio of minimum to average remaining battery capacity, and the network lifetime measured as the time when any node exhausts its battery capacity for the first time. Since these metrics provide limited information on energy balance, the expiration sequence gives more accurate information on how fairly energy is expended.

\subsection{Sleep/Power-Down Mode Approach}

Unlike the previous two subsections, the sleep/power-down mode approach shows its interest in inactive time of communication in nodes. Some applications will offer the nodes to take rest or sending data's periodically. Thus the radio will consume power without transmitting any data, so the nodes can be brought to sleep mode by switching off the radio when they are not transmitting any data to save energy. Even after if all the nodes in a MANET sleep then there is no node to listen, packets cannot be delivered to a destination node. One possible solution is to elect a special node, called a master, and let it coordinate the communication on behalf of its neighboring slave nodes. Now, slave nodes can safely sleep most of time saving battery energy. Each slave node periodically wakes up and communicates with the master node to find out if it has data to receive or not but it sleeps again if it is not addressed [5].

In a multihop MANET, more than one master node would be required because a single master have limited energy and cannot cover the entire MANET. Except Master nodes all other nodes can save energy by putting their radio hardware into low power state. The master-slave architecture is based on symmetric power model, where master nodes have the same radio power and thus the same transmission range as slave nodes. In the asymmetric power model, where master nodes have longer transmission range while this type of hierarchical network architecture has been actively studied for different reasons, such as interference reduction and ease of location management, the problem of selecting master nodes and maintaining the master-slave architecture under dynamic node configurations is still a challenging issue.

\subsection{GAF (Geographic Adaptive Fidelity) Protocol}

GAF protocol, [2] each node uses location information based on GPS to associate itself with a "virtual grid" so that the entire area is divided into several square grids, and the node with the highest residual energy within each grid becomes the master of the grid. The master node is selected based on hello packets exchanged between each other which carry the complete information including battery energy status. These data's will be loaded up in the routing table and the nodes which possess more energy will be chosen by all other nodes anonymously. Other nodes in the same grid can be regarded as redundant with respect to forwarding packets, and thus they can be safely put to sleep. The slave nodes switch between off and listening with the guarantee that one master node in each grid will stay awake to route packets. This incorporates the same procedure of Sleep/power-down approach after forming grid using GPS locations

\subsection{Energy Saving ES-AODV}

[7] AODV has a fixed transmission power. A transmission power control can be introduced to vary transmission power depending on the distance between the receiver and transmitter. The transmission power required can be calculated using the two ray propagation that was mentioned earlier in section 2.1. This is an easy improvement over AODV and does not cause problems. Routing discovery and route maintenance used here are same as that of in AODV.

\section{IMPLEMENTATION USING NS-2}

The software used is NS-2 as it contains a very strong energy module. ns is an object oriented simulator, written in $\mathrm{C}++$, with an OTcl interpreter as a frontend. The simulator supports a class hierarchy in $\mathrm{C}++$ (also called the compiled hierarchy in this document), and a similar class hierarchy within the OTcl interpreter (also called the interpreted hierarchy in this document). The two hierarchies are closely related to each other; from the user's perspective.

Ns uses two languages because simulator has two different kinds of things it needs to do [10].

- On one hand, a detailed simulation of protocols requires a systems programming language which can efficiently manipulate bytes, packet headers, and implement algorithms that run over large data sets. For these tasks run-time speed is important and turn-around time (run simulation, find bug, fix bug, recompile, re-run) is less important.

- $\quad$ On the other hand, a large part of network research involves slightly varying parameters or configurations, or quickly exploring a number of scenarios. In these cases, iteration time (change the model and re-run) is more important. Since 
configuration runs once (at the beginning of the simulation), run-time of this part of the task is less important.

\section{Portrait of A Mobile Node}

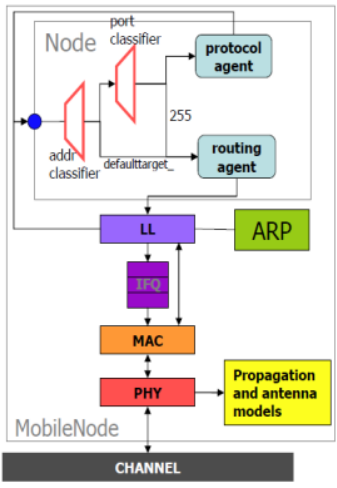

Classifier: Forwarding

Agent: Protocol Entity

- Node Entry

L. LL: Link layer object

IFQ: Interface queue

MAC: Mac object

PHY: Net interface

Figure 3. Structure of a node in NS2

\subsection{Modeling and Simulation parameters}

The below code describes the type of packet transfer protocol and its size.

set udp4_(0) [new Agent/UDP]

\$ns_attach-agent \$node_(0) \$udp4_(0)

set null4_(0) [new Agent/Null]

\$ns_attach-agent \$node_(8) \$null4_(0)

set cbr4_(0) [new Application/Traffic/CBR]

\$cbr4_(0) set packetSize_ 500

\$cbr4_(0) set interval_0.1

\$cbr4_(0) set random_1

\$cbr4_(0) set maxpkts_ 1000

\$cbr4_(0) attach-agent \$udp4_(0)

\$ns_connect \$udp4_(0) \$null4_(0)

\$ns_at 0.6 "\$cbr4_(0) start"

\$ns_at 5.5 "\$cbr4_(0) stop"

\section{Network 1:}

It is used for simulation of energy consumption of multiple source and single source scenarios.

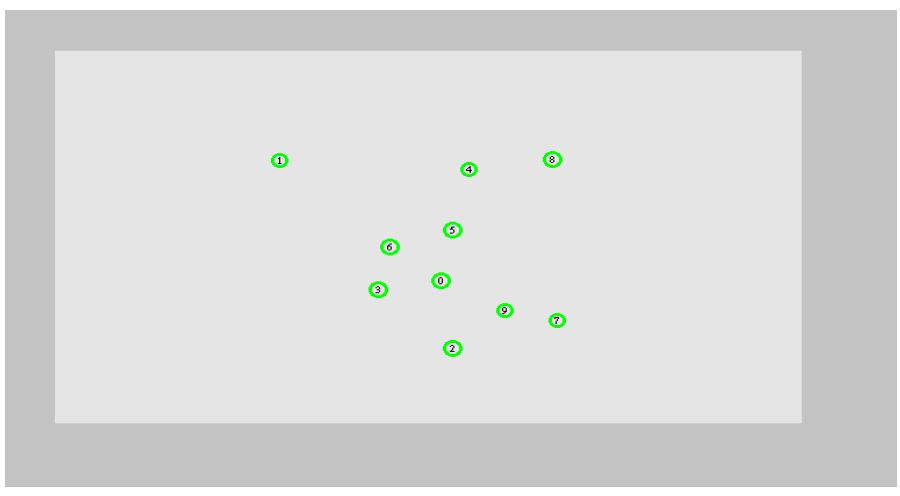

Figure 4. Wireless network with lesser nodes

Network 2

(11) (11)

(28)

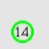

(4) (18) (24)

(3)

(21)

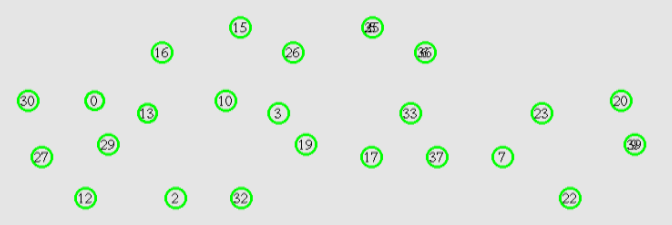

Figure 5.wireless network of 40 nodes

The above are created in NS2 and displayed using its animator NAM. It is used for simulation of packet flow using AODV in a Dense Network and High Traffic Scenario

\section{SIMULATION RESULTS}

\subsection{Performance Metrics}

The graph (Figure 6) was plotted from values obtained after simulation network1 with a single source using AODV and ES-AODV routing

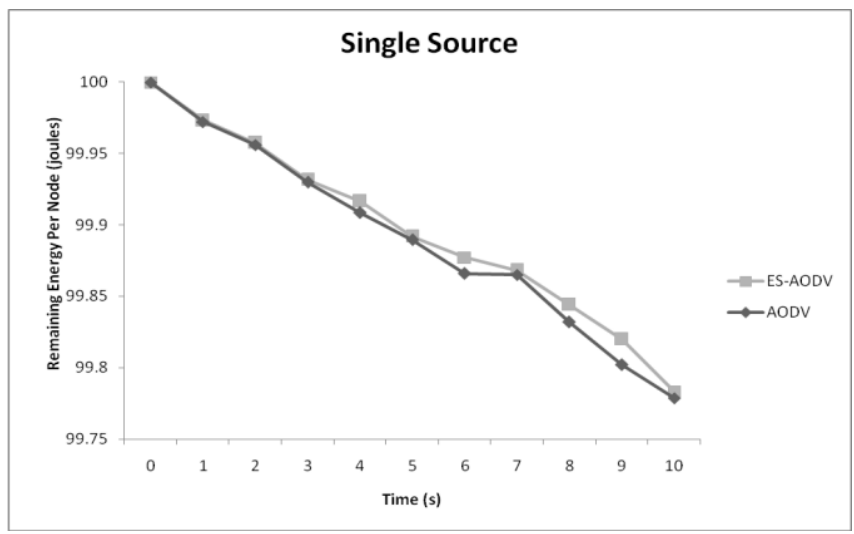

Figure 6 Shows graph plotted for single source

The graph shows (Figure 6) a small difference in the remaining energy per node, after packets were routed using 
AODV and ES-AODV routing. The average energy remaining per nodes at the end of simulation using AODV $=99.7786$ joules. The average energy remaining per node at the end of simulation using ES-AODV=99.7832 joules. Energy Conserved $=.0046$ joules

Graph drawn (Figure 7) from the values obtained after simulation for network 1 with multiple sources using AODV and ES-AODV routing.

From this graph we can see that the energy conserved is more as the number of sources in the network increases. Average Energy remaining per node after routing using AODV at the end of simulation $=99.4144832$ joules. Average Energy remaining per node after routing using ES-AODV at the end of simulation = 99.5013326 joules. Energy saved $=.0868494$ joules

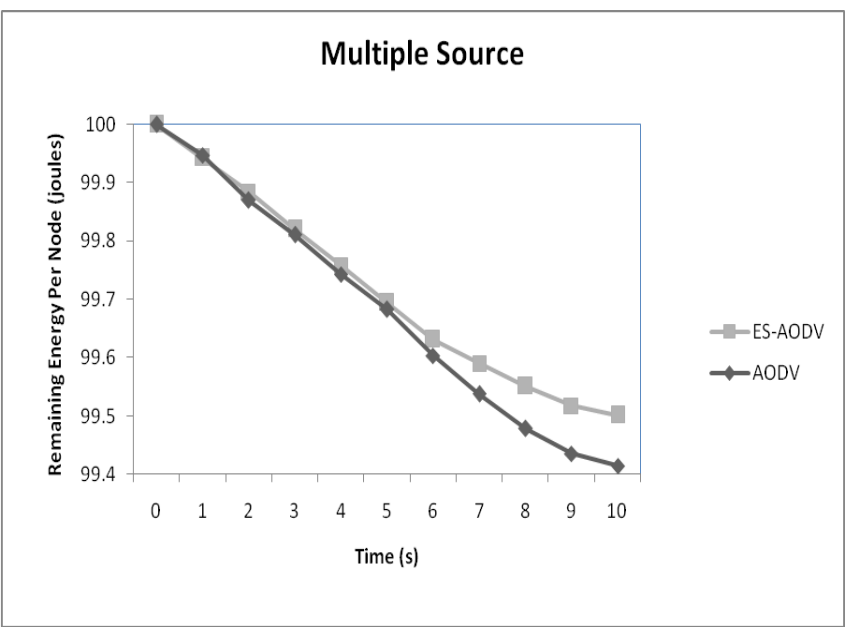

Figure 7. Shows graph plotted for multiple source

The graph (Figure 8) was plotted using values obtained after simulation of Network 2 with 40 nodes and multiple sources.

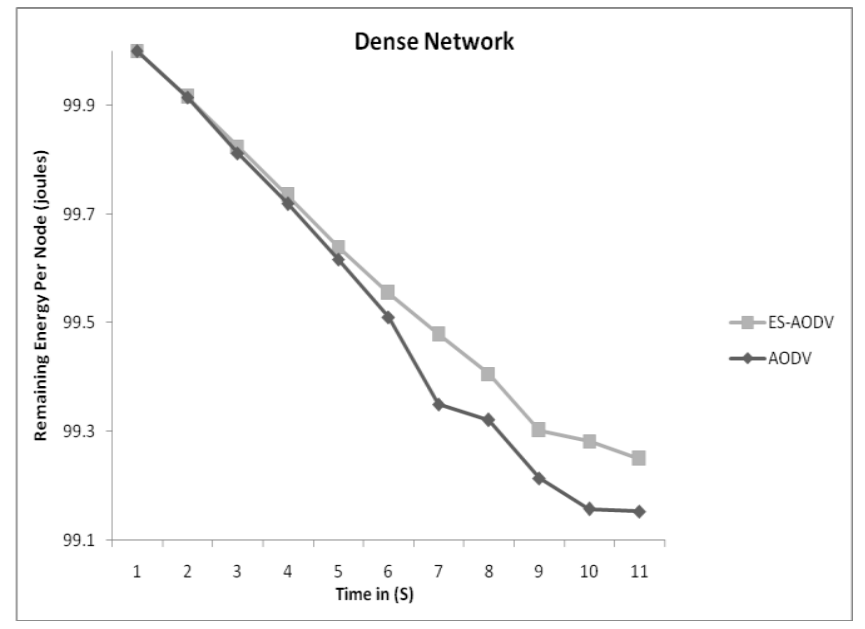

Figure 8. Shows graph plotted Dense Network

This graphs shows significant conservation of energy. This is because in a dense network internodal distance is less and as transmission power levels are set according to internodal distance in ES-AODV there is significant reduction in energy consumed for transmission. Average energy remaining per node after routing through AODV at the end of simulation = 99.1529 joules. Average energy remaining per node after routing through ES-AODV at the end of simulation $=99.25$ joules. Energy saved $=.3863$ joules

The graph (Figure 9) was plotted using values obtained after simulation of Network 2 with 40 nodes in a high traffic scenario.

The graph (Figure 9) shows that the average energy remaining in nodes after routing with $\mathrm{AODV}$ is less than quarter of the initial energy whereas average energy remaining in nodes with ES-AODV routing is more than half the initial energy. Initial energy is set to 2 joules. Average energy remaining per node after routing through AODV at the end of simulation $=.2616$ joules

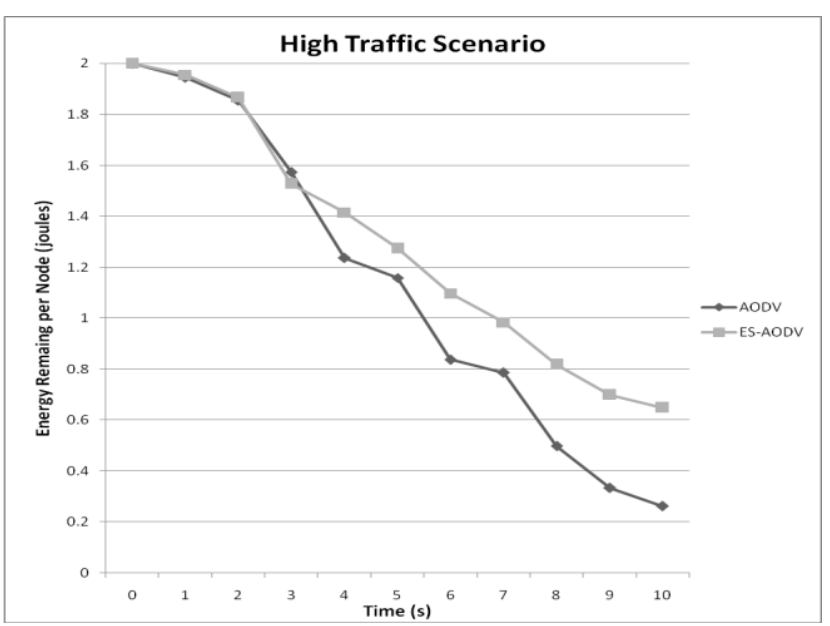

Figure 9. Shows graph plotted for multiple sources

Average energy remaining per node after routing through ES-AODV at the end of simulation $=.648$ joules. Energy conserved .386 joules. The figure 10 below shows the energy remaining in each node in condition described above

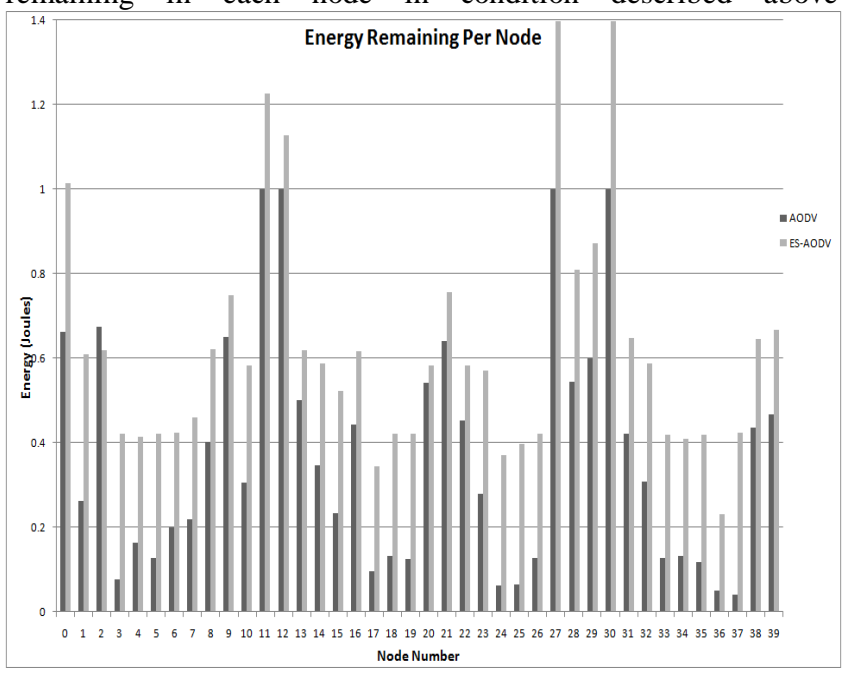

Figure 10. Shows Energy remaining per node

It shows that using AODV routing the network will soon will lose connectivity as more than 16 of its nodes are nearing 
death. While with ES-AODV all the nodes have connectivity and can continue operating for longer period of time

\subsection{Simulation Environment}

The images given below (figure 11) are images taken at the end of simulation. The packet size was set as 250, 500, 750 and 1000 and the simulation was run each time for AODV and ESAODV [10].

The green color on the node shows the nodes having most energy remaining. The yellow color on the nodes denotes the nodes that have used up a lot of their energy but has more than $25 \%$ of their initial energy, while the red color shows that the nodes have below $25 \%$ of their initial energy left and will soon die.
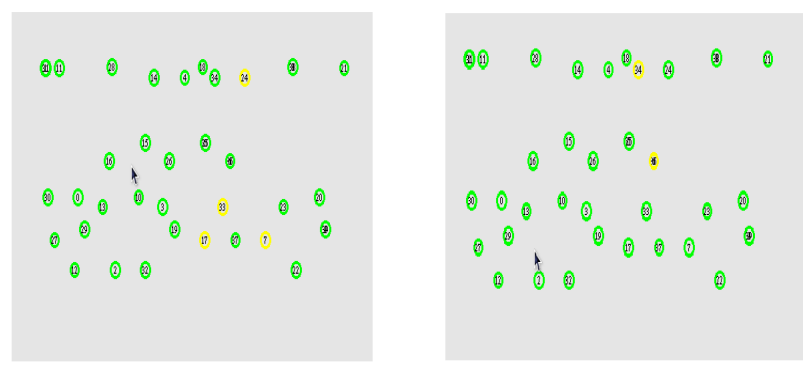

AODV with packet size 250

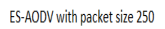

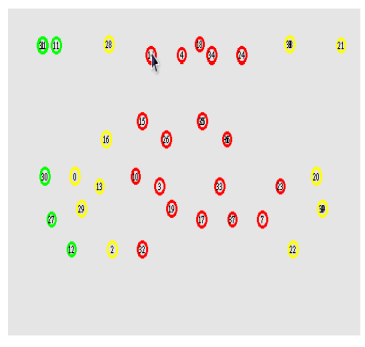

AOOV with packet size 500

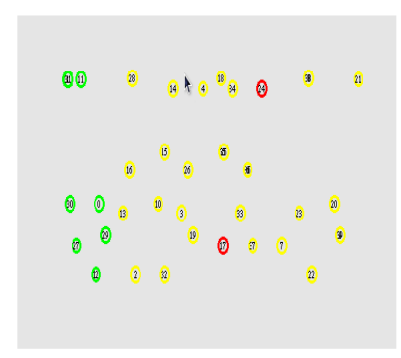

ES-A0DV with packet siee 500
Figure 11. Shows Snapshot after simulation with packet size 250 and 500

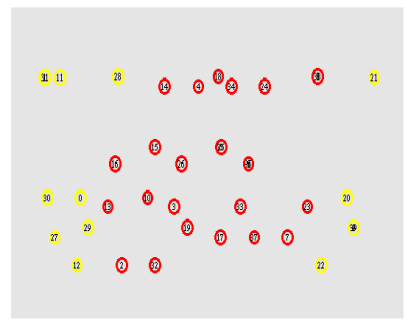

AODV with packet size 750

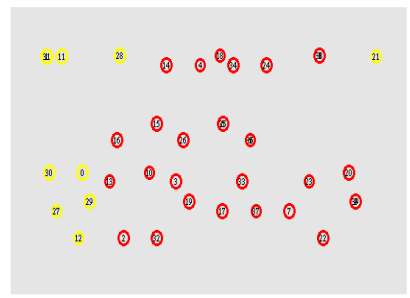

AODV with packet size 1000

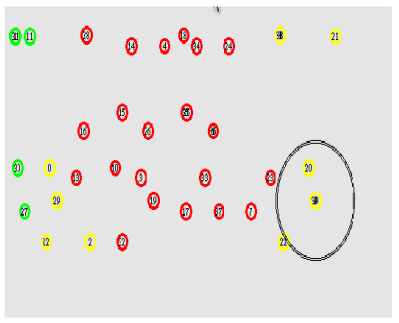

ES-AODV with packet size 750

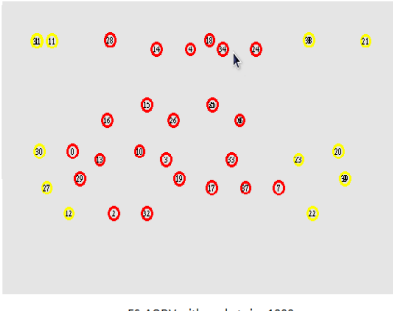

ES-AODV with packet size 1000
Figure 12. Shows Snapshot after simulation with packet size 750 and 1000

It can be understood from these diagrams that the ESAODV can save more power and therefore guarantees longer life for each node; which in-turn guarantees full connectivity for a longer time and increased operation time for the entire network.

\section{CONCLUSION}

In this simulation environment packets are forwarded between nodes using AODV routing protocols. But to decrease energy consumption and extend operation time of the network, transmission power control has been incorporated into AODV. The transmission power is varied according to distance between receiver and transmitter using two ray propagation models.

From the results it can be seen that the desired improvement over AODV has been attained. The networks using Energy Saving AODV are less energy consuming and proposing full connectivity over a longer period of time

\section{ACKNOWLEDGMENTS}

Our thanks to all the faculty colleagues who participated in discussions to improve the work and our VIT University for their continuous encouragement

\section{REFERENCES}

[1] "Energy Saving Routing Protocol Based on AODV" by Xingsheng Wang, Qing Liu. 2008

[2] Geography informed Energy Conservation, Ad Hoc Routing Seventh Annual ACM/IEEE International Conference on Mobile Computing and Networking(ACM Mobicom), July 1621, 2001, Rome, Italy

[3] $\mathrm{M}^{2}{ } \mathrm{Ri}^{2}$ : Multi-power, Multi-interface Routing Protocol for Intra/Inter-flow Interference Considerations in Wireless Mesh Networks, Tzu-Chieh Tsai, Sung-Ta Tsai, Fourth 
International Conference on Communications and Networking in China, 2009. China, 2009

[4] "Minimum energy mobile wireless networks," Volkan Rodoply and Teresa H. Meng, IEEE, vol17(8), 1999.

[5] "Optimizing Power Aware Routing in Mobile AdhocNetworks" by Jharna Chokhawala and Albert Mo Kim Cheng, 2001

[6] "Power-Aware Localized Routing in Wireless Networks", IEEE transactions on Parallel and Distributed Systems, Vol.12, no. 11, November 2001.
[7] 'Power and Placement: Increasing Mobile Ad-hoc Network Capacity and Power Efficiency', by RA Hunjet - 2009.

[8] Toh C-K. Maximum Battery Life Routing to Support Ubiquitous Mobile Computing in Wireless Ad Hoc Networks. IEEE Communications 2001.

[9] Woo K, Yu C, Youn HY, Lee B. Non-Blocking, Localized Routing Algorithm for Balanced Energy Consumption in Mobile Ad Hoc Networks. Proceedings of Int'l Symp. on Modeling, Analysis and Simulation of Computer and Telecommunication Systems (MASCOTS 2001) 2001;117124. 